\title{
LNK deficiency promotes acute aortic dissection and rupture
}

\author{
Fanny Laroumanie, ${ }^{1}$ Arina Korneva, ${ }^{2}$ Matthew R. Bersi, ${ }^{2,3}$ Matthew R. Alexander, ${ }^{4}$ Liang Xiao, ${ }^{1}$ \\ Xue Zhong, ${ }^{5}$ Justin P. Van Beusecum, ${ }^{1}$ Yuhan Chen, ${ }^{1}$ Mohamed A. Saleh, ${ }^{1},{ }^{12}$ William G. McMaster, ${ }^{6}$ \\ Kyle A. Gavulic, ${ }^{1}$ Bethany L. Dale, ${ }^{7}$ Shilin Zhao, ${ }^{8}$ Yan Guo, ${ }^{8}$ Yu Shyr, ${ }^{8}$ Daniel S. Perrien, ${ }^{1,9}$ \\ Nancy J. Cox, ${ }^{5}$ John A. Curci, ${ }^{10}$ Jay D. Humphrey, ${ }^{2}$ and Meena S. Madhur ${ }^{1,4,11}$ \\ 'Division of Clinical Pharmacology, Vanderbilt University Medical Center (VUMC), Nashville, Tennessee, USA. 2Department of \\ Biomedical Engineering, Yale University, New Haven, Connecticut, USA. ${ }^{3}$ Department of Biomedical Engineering, Vanderbilt \\ University (VU), Nashville, Tennessee, USA. ${ }^{4}$ Division of Cardiovascular Medicine, ${ }^{5}$ Division of Genetic Medicine, and ${ }^{6}$ Divison \\ of Thoracic Surgery, VUMC, Nashville, Tennessee, USA. 'Department of Molecular Physiology and Biophysics, VU, Nashville, \\ Tennessee, USA. ${ }^{8}$ Department of Biostatistics, ${ }^{9}$ Vanderbilt Center for Bone Biology, and ${ }^{10}$ Division of Vascular Surgery, \\ VUMC, Nashville, Tennessee, USA. "Vanderbilt Institute for Infection, Immunology and Inflammation, Nashville, Tennessee, \\ USA. ${ }^{12}$ Department of Pharmacology and Toxicology, Faculty of Pharmacy, Mansoura University, Mansoura, Egypt.
}

Aortic dissection (AD) is a life-threatening vascular disease with limited treatment strategies. Here, we show that loss of the CWAS-identified SH2B3 gene, encoding lymphocyte adaptor protein LNK, markedly increases susceptibility to acute $A D$ and rupture in response to angiotensin (Ang) II infusion. As early as day 3 following Ang II infusion, prior to the development of AD, $\mathrm{Lnk}^{-/-}$aortas display altered mechanical properties, increased elastin breaks, collagen thinning, enhanced neutrophil accumulation, and increased MMP-9 activity compared with WT mice. Adoptive transfer of $\mathrm{Lnk}^{-/-}$leukocytes into $\mathrm{Rag1}^{-/-}$mice induces $\mathrm{AD}$ and rupture in response to Ang II, demonstrating that LNK deficiency in hematopoietic cells plays a key role in this disease. Interestingly, treatment with doxycycline prevents the early accumulation of aortic neutrophils and significantly reduces the incidence of $A D$ and rupture. PrediXcan analysis in a biobank of more than 23,000 individuals reveals that decreased expression of $\mathrm{SH} 2 \mathrm{~B} 3$ is significantly associated with increased frequency of AD-related phenotypes (odds ratio 0.81 ). Thus, we identified a role for LNK in the pathology of AD in experimental animals and humans and describe a new model that can be used to inform both inherited and acquired forms of this disease.

Conflict of interest: MSM received a research grant from Gilead Sciences, Inc.

License: Copyright 2018, American Society for Clinical Investigation.

Submitted: May 30, 2018

Accepted: August 30, 2018

Published: October 18, 2018

\section{Reference information:} JCI Insight. 2018;3(20):e122558. https://doi.org/10.1172/jici. insight.122558.

\section{Introduction}

Aortic dissection (AD) is an acute and often lethal disease characterized by disruption in the medial layer of the aortic wall resulting in formation of a false lumen and/or intramural hematoma. Subsequent compression of the true lumen may result in compromised end-organ perfusion, and loss of structural wall integrity can result in fatal aortic rupture. $\mathrm{AD}$ should be differentiated from aortic aneurysm, which is defined as a focal dilation of the vessel wall to more than $50 \%$ of the normal diameter (1). Optimal treatment strategies for $\mathrm{AD}$ are limited, likely due to poor insight into its pathogenesis and the paucity of suitable animal models to investigate early stages of disease and response to therapy.

Major risk factors for $\mathrm{AD}$ include inherited connective tissue diseases, male gender, hypertension, and autoimmune vascular diseases $(1,2)$. Importantly, growing evidence suggests that inflammation contributes to the development of AD. Inflammatory cells have been found in dissected aortic walls of humans and experimental animals. It has been hypothesized that these cells secrete MMPs and release reactive oxygen species that could induce medial degradation and predispose to dissection (3). Furthermore, inflammatory biomarkers are often elevated in the blood of patients with AD and correlate with disease activity (3). However, major unresolved questions include which immune cells and pathways are the key players and whether these cells are a cause or consequence of $\mathrm{AD}$.

Multiple GWAS in humans reveal that a missense SNP in the SH2B3 gene, encoding the lymphocyte adaptor protein LNK, is associated with several autoimmune and cardiovascular disorders, including lupus and hypertension (4). LNK is expressed in all hematopoietic cells and endothelial cells and functions as a 
negative regulator of cytokine signaling and cell proliferation. We previously showed that LNK deficiency promotes hypertension and vascular inflammation (5).

Here, we report the finding that $L n k^{-/}$mice are predisposed to the development of acute $\mathrm{AD}$ and rupture, independent of BP. As early as 3 days following angiotensin (Ang) II infusion (prior to the development of $\mathrm{AD}), \mathrm{Lnk}^{-1-}$ aortas exhibit altered mechanical properties, primarily in the suprarenal abdominal aorta (SAA), corresponding with adverse extracellular matrix remodeling, increased neutrophils, and enhanced MMP-9 activity. Furthermore, we found that neutrophils from $L n k^{-1-}$ mice exhibit enhanced migration and increased MMP-9 production when stimulated ex vivo. Inhibition of MMPs and aortic neutrophil accumulation with doxycycline significantly reduced the incidence of $\mathrm{AD}$ in $\mathrm{Lnk}^{-/-}$mice. Interestingly, PrediXcan analysis in a biobank of more than 23,000 individuals reveals that decreased expression of $S H 2 B 3$ is significantly associated with increased frequency of AD-related phenotypes. Taken together, our study highlights a key role for LNK in experimental and human $\mathrm{AD}$ and provides a potentially unique and novel model to investigate mechanisms of $\mathrm{AD}$.

\section{Results}

LNK deficiency increases susceptibility to AD and rupture in Ang II-infused mice. To determine the role of LNK in the development of $\mathrm{AD}$, we infused male $L n k^{-/-}$and WT control mice with Ang II $(1,200 \mathrm{ng} / \mathrm{kg} / \mathrm{min})$ for 14 days. Interestingly, $63 \%$ of male $L n k^{-1-}$ mice developed $\mathrm{AD}$ or fatal aortic rupture, primarily in the SAA, compared with $18 \%$ of male WT mice (Figure 1, A and B). BP and plasma cholesterol were similar between Ang II-infused $\mathrm{Lnk}^{-/}$and WT mice (Supplemental Figure 1, A and B; supplemental material available online with this article; https://doi.org/10.1172/jci.insight.122558DS1). Dissected Lnk ${ }^{-/}$aortas had a compressed true lumen as well as either an intramural hematoma or a large false lumen with thrombosed blood (Figure 1C). The primary immune cells at the site of dissection were neutrophils and macrophages (Figure 1D) with few T and B cells (Supplemental Figure 1C). Interestingly, histopathological examination of the abdominal aortas from mice that survived 14 days of Ang II infusion revealed differences in aortic remodeling between WT and $L n k^{-/-}$mice. WT abdominal aortas exhibited intense adventitial fibrosis and preservation of elastic fiber integrity, whereas $L n k^{-1-}$ abdominal aortas displayed less fibrosis and signs of elastin disruption (Figure 1E). To track changes in luminal diameter and inflammatory cell infiltration in live animals, we performed microCT scanning using gold nanoparticles that target uptake by phagocytic cells. Luminal diameter showed no significant change during the course of Ang II infusion in a representative $L n k^{-/-}$mouse. Phagocytic inflammatory cells appeared as early as day 3 and were localized to the celiac and superior mesenteric artery branches (Supplemental Figure 1D). Consistent with male predominance of $\mathrm{AD}$ seen in humans (2), female $L n k^{-/}$and WT mice were largely protected from AD, despite similar BPs, with only $7 \%$ of $L n k^{-1-}$ females and no WT females developing the disease (Figure $1 \mathrm{~F}$ and Supplemental Figure 2). Thus, LNK deficiency increases susceptibility to AD and rupture in a manner that phenocopies the human disease, including the feature of male predominance. Due to this gender difference, the remainder of the studies described here were conducted in male mice.

LNK deficiency promotes early changes in mechanical and structural properties of the SAA. To determine if early differences in the mechanical properties of $L n k^{-/}$aortas could predispose to AD, we performed biaxial mechanical tests on aortas from WT and $L n k^{-1-}$ mice infused with vehicle (sham) or Ang II for 3 days. Mice that developed $\mathrm{AD}$ at the time of or prior to euthanasia were excluded from this analysis. When compared at group-specific BPs and in vivo axial stretches, there were no baseline differences in mechanical properties of the ascending thoracic aorta, descending thoracic aorta, SAA, or infrarenal abdominal aorta from WT and $L n k^{-/}$mice. However, after 3 days of Ang II infusion, the greatest differences in mechanical properties between $L n k^{-/-}$and WT mice were found in the SAA (Figure 2, A-D, and Supplemental Tables 1-4). Ang II infusion resulted in a significant increase in outer diameter and stored energy of the SAA in WT mice, but these changes were attenuated in $L n k^{-/-}$mice (Figure 2 , A and B). In the absence of any change in wall thickness between the groups (Supplemental Table 3), these data suggest decreased distensibility of the SAA in $L_{n k^{-1-}}$ mice. Ang II increased circumferential stress and stiffness in both groups of mice, though to a lesser extent in Lnk $^{-/-}$compared with WT mice (Figure 2, C and D), despite similar BPs. Importantly, we also observed several instances of mechanical failure (i.e., intramural delamination) in the SAA from $L n k^{-1-}$ Ang II-treated mice during the initial stages of the biaxial mechanical testing protocol at physiological loads compared with only 1 late transverse medial tear in WT Ang II-treated mice (Supplemental Figure 3A). 
A

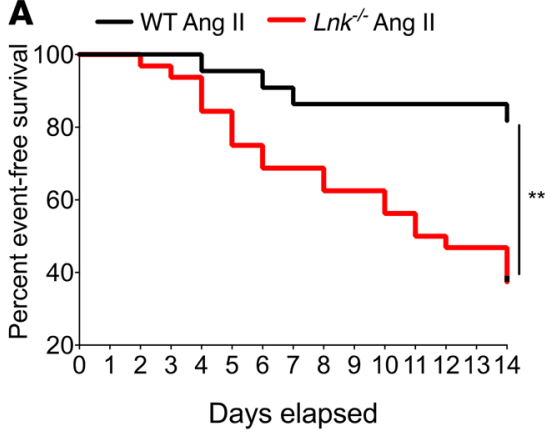

D

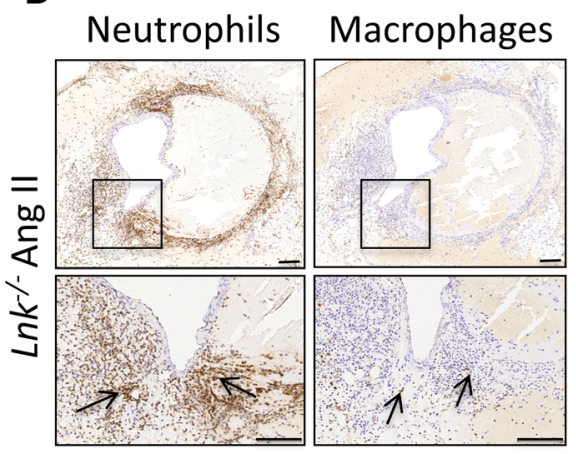

F $\quad$ - Female WT Ang II - Female LnK ${ }^{/-}$Ang II

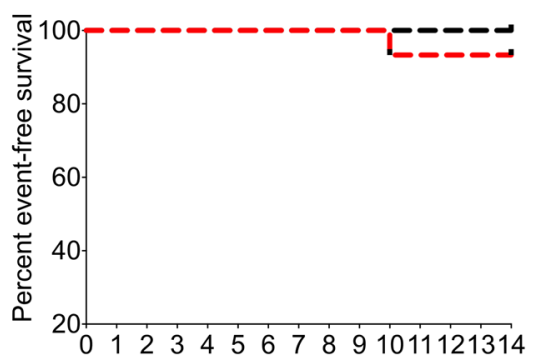

Days elapsed
B

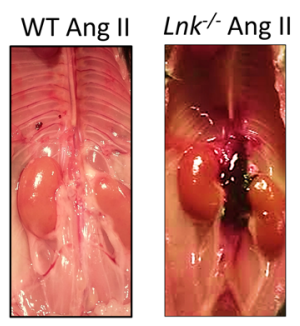

C

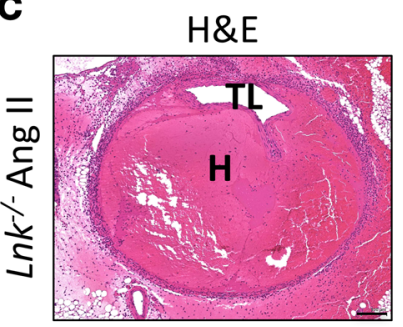

E

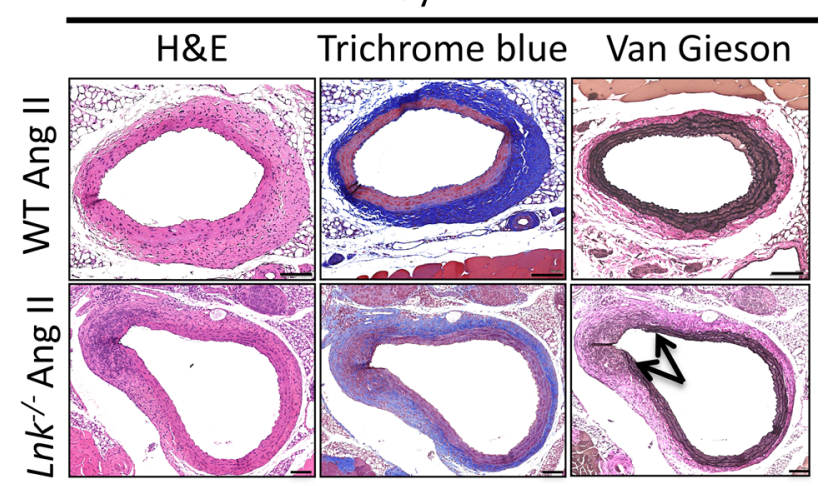

Figure 1. LNK deficiency predisposes to aortic dissection (AD) and rupture in angiotensin (Ang) II-infused mice. (A) Kaplan-Meier survival curves of male WT and $\mathrm{Lnk}^{-/-}$mice during 14 days of Ang II infusion $(n=22-32)$. (B) Representative gross images of Ang II-infused WT and $\mathrm{Lnk}^{-1-}$ aortas showing rupture and hematoma in the abdominal aorta. (C) Representative transverse section of dissected abdominal aorta from Ang II-infused $\mathrm{LnK}^{-1-}$ mice stained with hematoxylin and eosin (H\&E). $\mathrm{TL}$, true lumen; $\mathrm{H}$, hematoma. (D) Representative immunohistochemical staining for neutrophils (Ly-6C/-6C) and macrophages (F4/80) in the dissected abdominal aorta of Ang II-infused $\mathrm{Lnk}^{-1-}$ mice. Scale bars: $100 \mu \mathrm{m}$ (C and D). (E) Representative transverse sections of abdominal aorta from Ang II-infused WT and $\mathrm{LnK}^{-1-}$ mice stained with $\mathrm{H} \& \mathrm{E}$, Masson's trichrome blue, and Elastica van Gieson. Arrows depict site of elastin degradation. Scale bars: $100 \mu \mathrm{m}$. (F) Kaplan-Meier survival curves of female WT and $L n k^{-1-}$ mice during 14 days of Ang II infusion $(n=7-15)$. ${ }^{* *} P<0.01$ by Mantel-Cox test.

We then sought to determine if these mechanical alterations correlated with microstructural changes. After 3 days of Ang II infusion, there were 2-fold more elastin breaks in the abdominal aorta of $L^{n k^{-1}}$ mice compared with WT mice, and the elastic fibers of $\mathrm{Lnk}^{-1-}$ mice exhibited a loss of the spring-like elastic lamellae structures (Figure 2E). These changes are consistent with the observed reduction in energy storage capacity of $L n k^{-1}$ SAAs. Ang II infusion also increased fibrosis and collagen deposition in the abdominal aortas of WT mice, and these changes were significantly attenuated in $\mathrm{Lnk}^{-1}$ mice (Figure $2 \mathrm{~F}$ and Supplemental Figure 3B). Picrosirius red staining revealed similar collagen fiber organization and thickness (reflected by similar percentages of red, orange, yellow, and green fibers) in the abdominal aortas of WT and $L n k^{-/-}$mice at baseline. Strikingly, after 3 days of Ang II infusion, these fibers appeared less densely packed with fewer thick fibers (red) and more thin fibers (orange, yellow, and green) in $L n k^{-1}$ compared with WT mice (Figure 2G). Of note, there was no change in the mRNA expression for the major procollagens, Colla1 and Col3a1, supporting a role for collagen degradation rather than decreased production in $L n k^{-1-}$ mice (Supplemental Figure 3C). These results demonstrate that prior to the development of $\mathrm{AD}$, there are early microstructural and mechanical changes in the aortas of $L n k^{-1}$ mice that, in the absence of a protective fibrotic adventitia, likely resulted in increased vulnerability to rupture.

LNK deficiency induces early MMP-9 activity in the abdominal aorta prior to the development of $A D$. MMPs, particularly MMP-2 and MMP-9, have been widely implicated in aortic disease. By quantitative reverse transcription PCR (qRT-PCR) on aortas from $L n k^{--}$and WT mice infused with Ang II for 3 days, we detected increased expression of MMP-2 and MMP-9 in $\mathrm{Lnk}^{-/-}$mice, with no change in expression of the tissue 


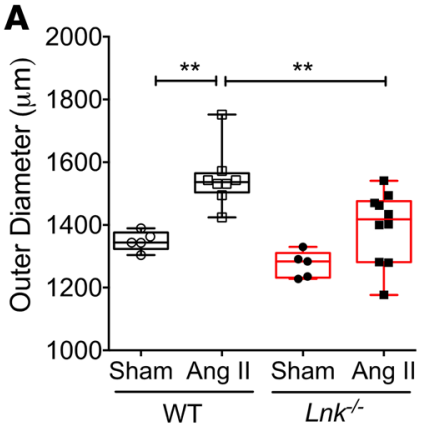

E WT Ang II LnK ${ }^{\prime-}$ Ang II
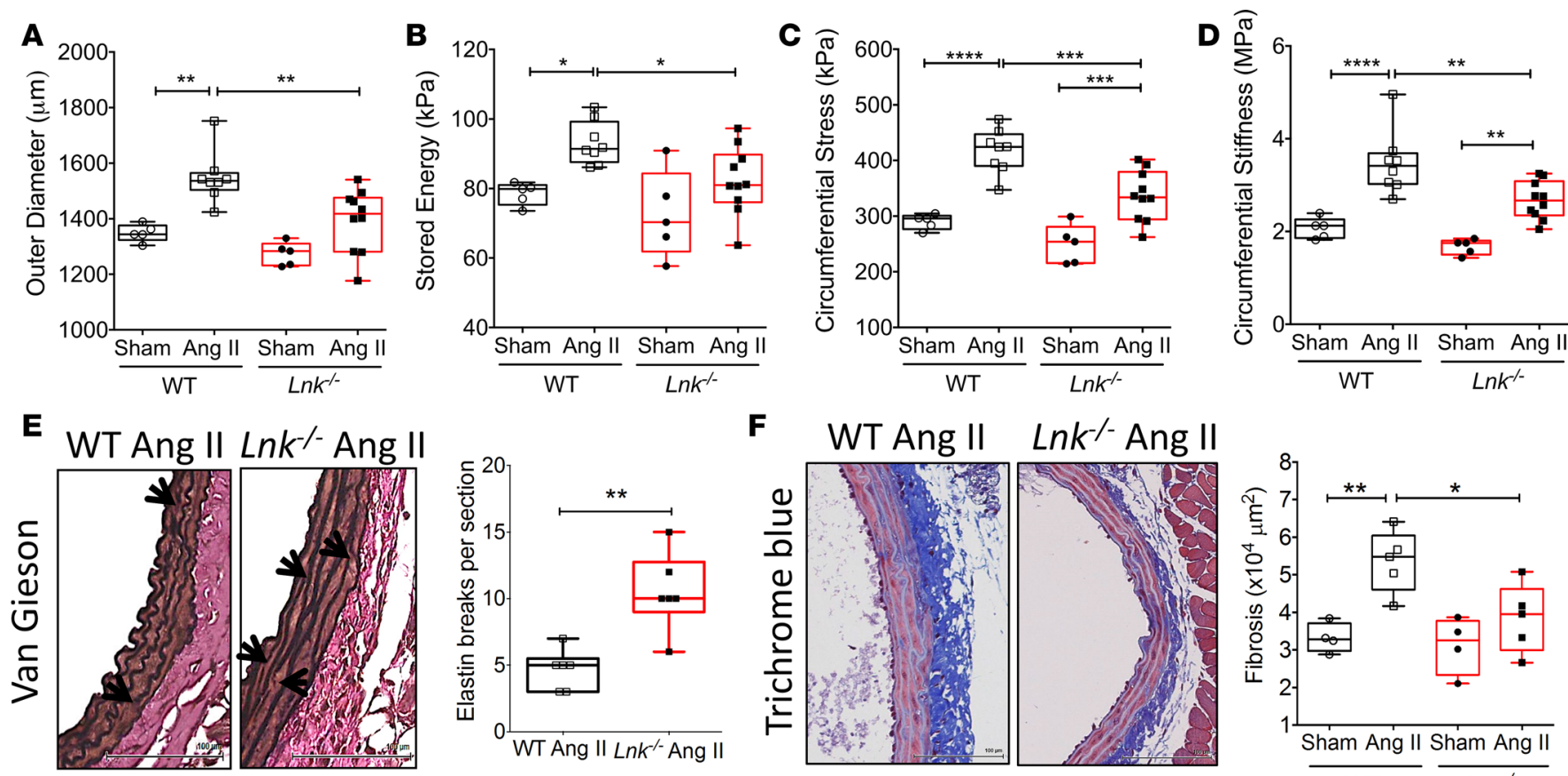

F WT Ang II Lnk ${ }^{-1}$ Ang II
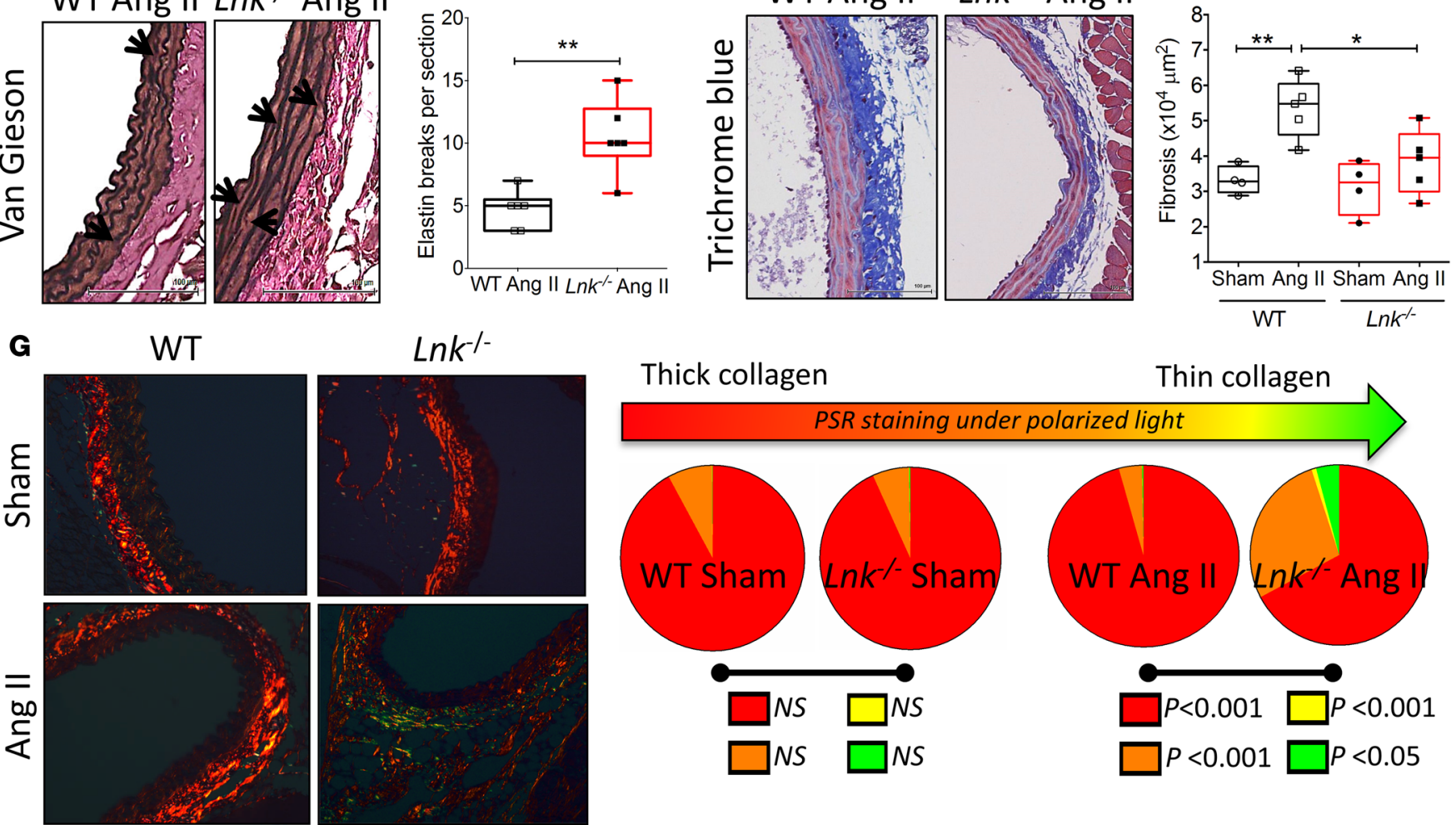

Thick collagen

\section{Thin collagen}

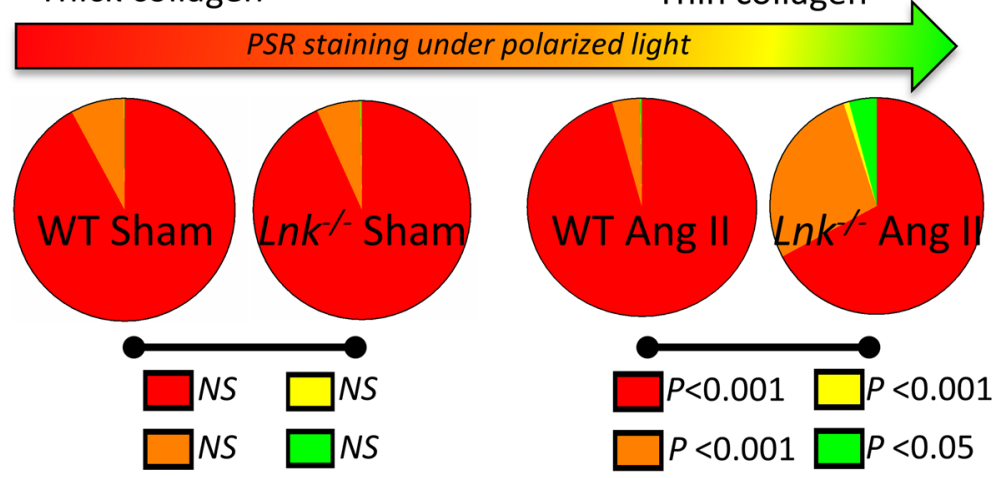

Figure 2. LNK deficiency promotes early changes in mechanical and structural properties of the suprarenal abdominal aorta. WT and $L n k^{-/-}$mice were infused with vehicle (sham) or Ang II for 3 days. (A) Outer diameter, (B) stored energy capacity, (C) circumferential stress, and (D) circumferential stiffness measured in vitro via biaxial tests in the indicated groups at group specific systolic pressures and in vivo axial stretches ( $n=5-10)$. (E) Representative Elastica van Gieson staining of the abdominal aorta. Arrows indicate elastin breaks, which are quantified on the right ( $n=6)$. (F) Representative Masson's trichrome staining of the abdominal aorta with quantification of fibrosis shown on the right $(n=4-5)$. (G) Picrosirius red (PSR) staining of abdominal aortic sections visualized under polarized light. Red indicates thick collagen fibers, while orange/yellow/green indicate progressively thinner collagen fibers. Representative images are shown on the left. Quantification of percentage of each color is shown on the right as pie charts ( $n=3-6$ ). Data are expressed as box-and-whisker plots $(\mathbf{A}-\mathbf{F})$. ${ }^{*} P<0.05$, ${ }^{* *} P<0.01$, ${ }^{* *} P<0.001,{ }^{* * *} P<0.0001$ by 2 -way ANOVA (A-D, and F) or by 2 -sided Student's $t$ test (E). For panel G, 2-way ANOVA was performed and $P$ values for each color are shown for the indicated comparisons. NS, nonsignificant.

inhibitors of metalloproteinases TIMP-1 and -2 (Figure 3A). Gelatin zymography revealed a slight increase in aortic MMP-2 activity in both WT and $\mathrm{Lnk}^{-1-}$ mice in response to Ang II infusion, which reached significance only in WT mice (Figure 3B). In contrast, the aortas of $L n k^{-1-}$ mice displayed a striking increase in MMP-9 activity with Ang II infusion that exceeded levels in Ang II-treated WT mice (Figure 3B). These results suggest that in the absence of LNK, increased MMP-9 activity leads to matrix degradation in the abdominal aorta that predisposes to the development of $\mathrm{AD}$.

$L N K$ deficiency in hematopoietic cells increases susceptibility to $A D$. To assess the role of hematopoietic cells in $\mathrm{AD}$, we adoptively transferred CD45+ leukocytes from WT or $L n k^{-1-}$ mice into $R a g 1^{-/-}$mice, which lack T and B cells. Interestingly, following Ang II infusion, $36 \%$ of $R a g 1^{-1-}$ mice that received $L n k^{-1-} \mathrm{CD} 45^{+}$cells died from $\mathrm{AD}$ and rupture compared with $0 \%$ of $\mathrm{Rag}^{1^{--}}$mice that received WT CD $45^{+}$cells (Figure 4). Further 
A

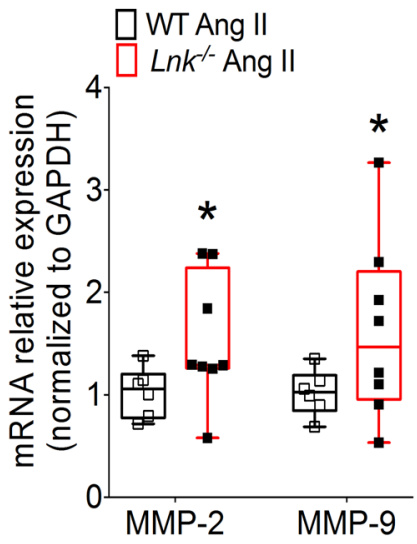

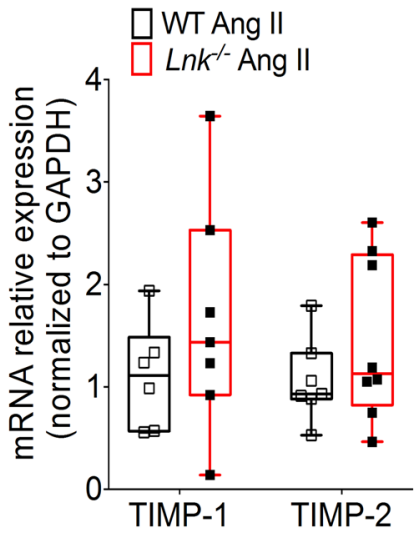
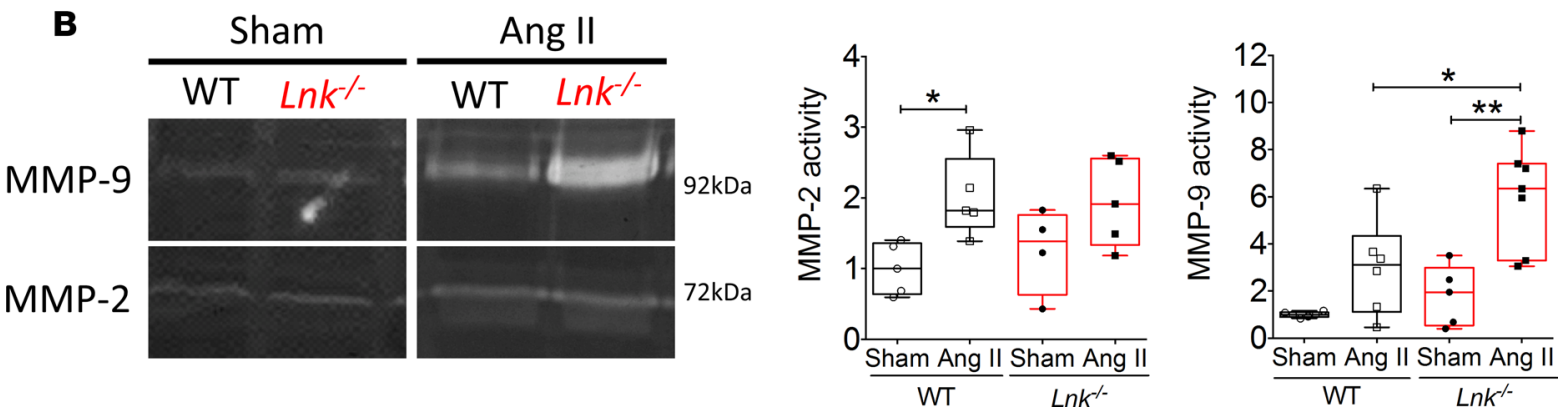

Figure 3. LNK deficiency induces early MMP-9 activity in the abdominal aorta prior to the development of AD. (A) Aortic mRNA expression of MMP-2, MMP-9, and tissue inhibitor of metalloproteinases 1 and 2 (TIMP-1 and -2) from WT and $L n k^{-1-}$ mice infused for 3 days with Ang II. mRNA expression was normalized to GAPDH and is represented as fold change relative to WT Ang II group $(n=6-8)$. (B) Representative gelatin zymograms of MMP-2 and MMP-9 activity in abdominal aortic protein lysates. Quantification (expressed as fold increase relative to WT sham) is shown on the right $(n=4-7)$. Data are expressed as box-and-whisker plots. ${ }^{*} P<0.05,{ }^{* *} P<0.01$ by 1 -sided Student's $t$ test (A) or by 2-way ANOVA (B).

evidence that immunological alterations in $\mathrm{Lnk}^{-/}$mice contribute to AD comes from RNA sequencing and KEGG pathway analysis of aortic tissue from WT and $L n k^{-/}$mice following 3 days of Ang II infusion, which revealed differential expression of genes enriched in multiple immune-related pathways such as graft-versushost disease and hematopoietic cell lineage (Supplemental Figure 4, A and B).

$L N K$ deficiency promotes early neutrophil recruitment in the aortic wall prior to the development of $A D$. Flow cytometry performed on aortic single-cell suspensions from $L n k^{-/}$and WT mice after 3 days of vehicle (sham) or Ang II infusion revealed a trend for increased neutrophils in WT mice that did not reach significance. In contrast, $\mathrm{Lnk}^{-1-}$ aortas displayed a marked increase in neutrophil accumulation that exceeded levels in Ang II-treated WT mice (Figure 5, A and B, and Supplemental Figure 5). Macrophage accumulation increased similarly with Ang II infusion in both groups (Figure 5B), and no differences were observed in the number of aortic M1-like or M2-like macrophage subsets between Ang II-infused WT and Lnk ${ }^{-1}$ mice (Supplemental Figure 6). By immunohistochemistry, neutrophils were scant in the abdominal aorta of Ang II-treated WT mice, but were prominent and present in all 3 layers (intima, media, and adventitia) of the aortic wall in Ang II-treated $L_{n k^{-1-}}$ mice (Figure 5C). The abdominal aortas of WT and $L n k^{-1-}$ mice treated with Ang II exhibited similar accumulation of macrophages, which occurred primarily in the adventitia (Figure 5C). At this early time point, there was no difference in aortic T or B cells in any of the 4 groups (Supplemental Figure 7, A and B). Thus, early aortic neutrophil recruitment in $\mathrm{Lnk}^{-1-}$ mice precedes the development of $\mathrm{AD}$.

To determine whether enhanced aortic chemokine expression is involved in the increased neutrophil recruitment in $\mathrm{Lnk}^{-1-}$ mice, we first performed qRT-PCR for the neutrophil-attracting chemokines CXCL1 and CXCL2 in WT and $\mathrm{Lnk}^{--}$aortas following 3 days of Ang II infusion and observed no difference (Supplemental Figure 8A). We also quantified additional chemokine protein levels using a chemokine array, which revealed no major differences in aortic chemokine expression between WT and $L n k^{-/-}$aortas following Ang II infusion (Supplemental Figure 8B). 


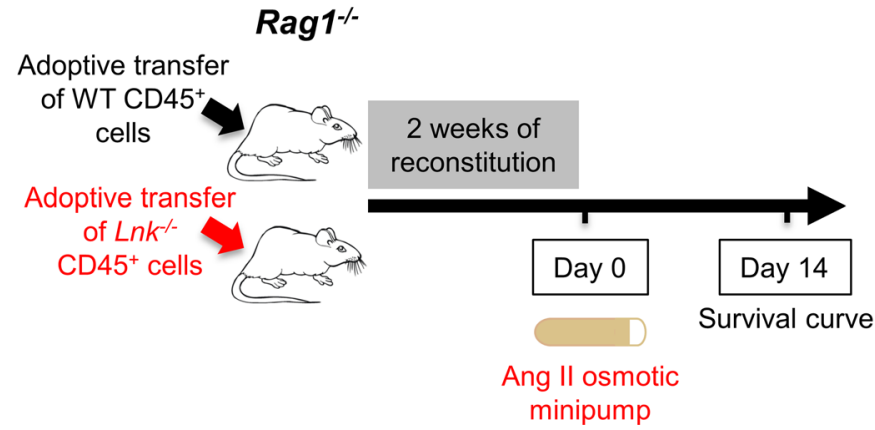

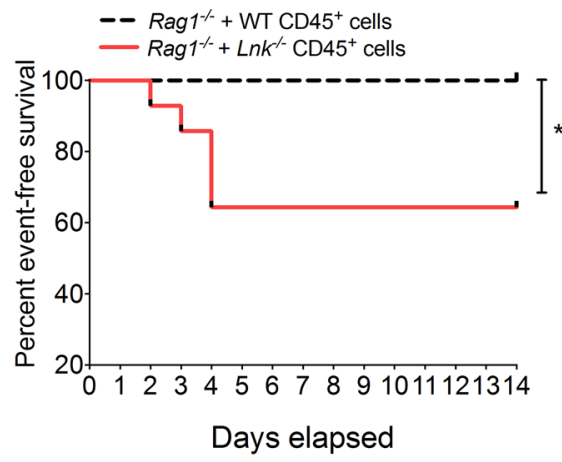

Figure 4. Loss of LNK in hematopoietic cells increases susceptibility to AD. Experimental design for adoptive transfer of CD45 leukocytes from WT or $\mathrm{Lnk}^{-/-}$mice into $\mathrm{Rag}^{-/-}$mice followed by Ang II infusion. Kaplan-Meier survival curves of the indicated groups during 14 days of Ang II infusion is shown on the right $(n=10-14) .{ }^{*} P<0.05$ by Mantel-Cox test.

We then investigated whether LNK-deficient neutrophils display an increased migratory capacity when exposed to CXCL2 in a transwell assay. Neutrophils were isolated from the bone marrow of WT and Lnk mice at baseline and after 3 days of Ang II infusion. The number of migrated neutrophils was the same between WT and $\mathrm{Lnk}^{-/-}$mice at baseline (Figure 5D). Interestingly, neutrophils from Ang II-infused mice exhibited increased migration, and this increase was significantly greater in $L n k^{-/}$mice compared with WT mice (Figure 5D). This enhanced migration was independent of the expression of the chemokine receptors CXCR1 and CXCR2, since qRT-PCR analysis demonstrated similar expression levels of these receptors in bone marrow-derived neutrophils from Ang II-infused WT and $L n k^{-1-}$ mice (Supplemental Figure 9). Since MMP-9 activity plays a key role in neutrophil tissue migration, we then examined MMP-9 activity from bone marrow-derived neutrophils and found that $\mathrm{Lnk}^{-1-}$ neutrophils indeed release more MMP-9 when activated ex vivo (Figure 5E). Lnk ${ }^{-/-}$mice have increased circulating numbers of most hematopoietic cells, including neutrophils (data not shown). Together, these data suggest that the combination of increased circulating neutrophil numbers, increased migratory capacity, and increased MMP-9 production results in the increased aortic neutrophil recruitment in $L n k^{-/}$mice.

Inhibition of neutrophil infiltration and MMP activity by doxycycline markedly reduced susceptibility to $A D$ and rupture in $\mathrm{Lnk}^{-1-}$ mice. The tetracycline antibiotic doxycycline potently inhibits a broad range of MMPs by binding the zinc-containing active site (6) and inhibits neutrophil activity and migration (7, 8). To test the hypothesis that both neutrophils and MMP activity are important in the development of $\mathrm{AD}$, we treated $L n k^{-1-}$ mice with doxycycline at $100 \mathrm{mg} / \mathrm{kg} /$ day, a dose that achieves plasma levels in mice comparable to levels in humans treated with doxycycline (9), prior to and during Ang II infusion (Figure 6A). Following 3 days of Ang II infusion, doxycycline treatment modestly lowered the percentage of circulating neutrophils (Figure 6B) and markedly reduced the accumulation of aortic neutrophils (Figure 6C) in $L n k^{-/}$mice. Strikingly, treatment of $L n k^{-1-}$ mice with doxycycline significantly reduced the incidence $\mathrm{AD}$ or fatal aortic rupture from $63 \%$ to $31 \%$ (Figure $6 \mathrm{D}$ ). Immunohistochemistry demonstrated reduced fibrosis and preserved elastin integrity in the abdominal aortas of $L n k^{-1-}$ mice treated with doxycycline that survived to day 14 of Ang II infusion (Figure 6E).

Reduced SH2B3 expression is associated with increased risk of AD-related phenotypes in humans. To determine the relevance of LNK expression to human AD, we used PrediXcan. This is a recently developed method that uses gene expression prediction models built from genome-transcriptome data sets such as the Genotype-Tissue Expression (GTEx) Project to predict gene expression from genotype and then tests the predicted expression levels for association with a phenotype of interest $(10,11)$. The $\mathrm{AD}$ phenotype was defined as containing at least one of the following ICD9 codes: 441, 441.00, 441.01, 441.02, 441.03; and the controls were defined as not having any diseases of the arteries, arterioles, and capillaries (i.e., excluding phecodes 440-449.99; https://phewascatalog.org/phecodes). We found that in 23,249 individuals of European ancestry, a combination of 32 SNPs that predict SH2B3 expression (Supplemental Table 5) was associated with AD-related ICD9 codes (odds ratio $[\mathrm{OR}]=0.81, P=0.0041$ ) (Figure 7). The OR less than 1 implies that reduced $S H 2 B 3$ expression is associated with higher risk of AD. 


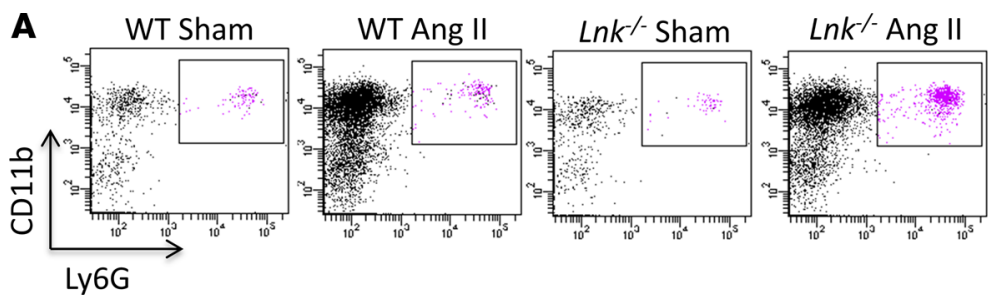

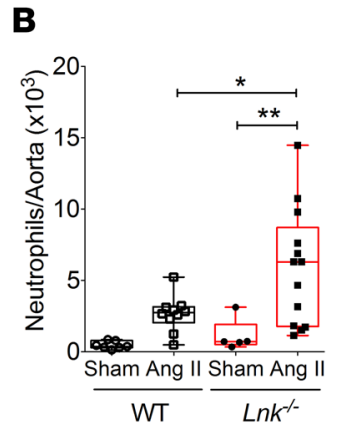

D

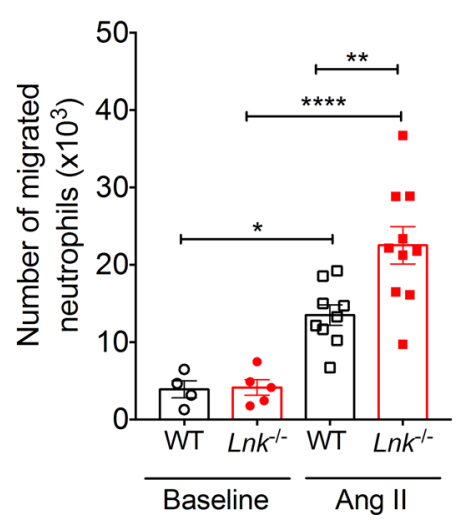

C Neutrophils
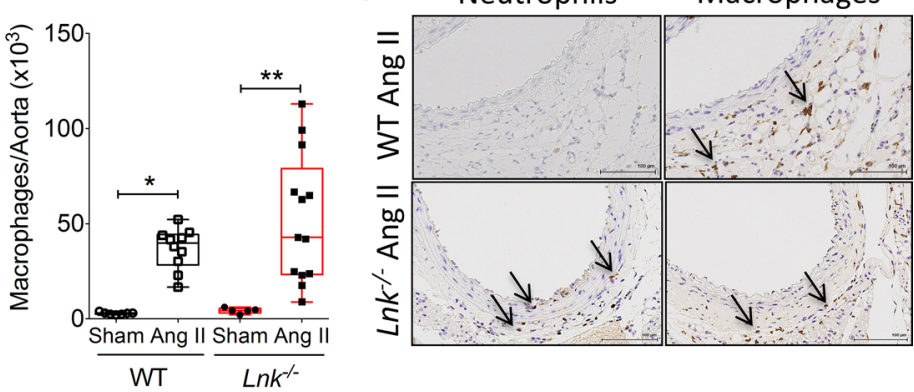

$\mathbf{E}$

WT

MMP-9
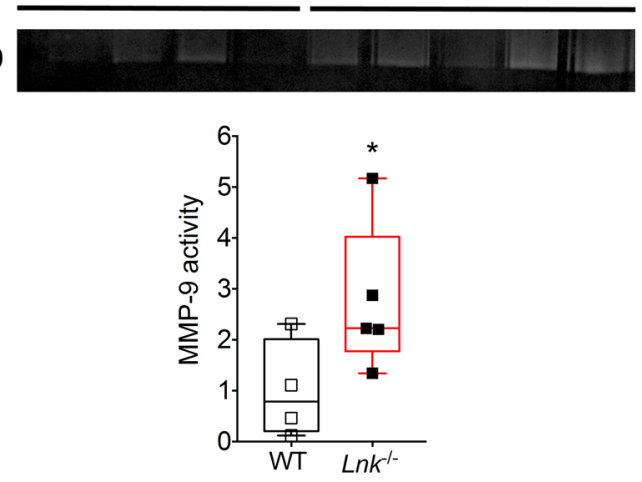

Figure 5. LNK deficiency promotes aortic neutrophil migration. (A) Representative flow cytometry dot plots of neutrophils in the aorta of WT and $\mathrm{Lnk}^{-/-}$mice infused with vehicle (sham) or Ang II for 3 days. Cells were gated from CD45 cells. (B) Flow cytometric quantification of neutrophils (CD45+CD11b $\left.{ }^{+} \mathrm{Ly} 6 \mathrm{C}^{+}\right)$and macrophages (CD45+CD11b $\mathrm{b}^{+} \mathrm{F} 4 / 80^{+}$cells) in the aorta of WT and $L n k^{-1-}$ mice infused with vehicle (sham) or Ang II for 3 days $(n=5-13)$. (C) Representative immunohistochemical staining for neutrophils (Ly-6C/-6C) and macrophages (F4/80) in the abdominal aorta of WT and $\mathrm{Lnk}^{-1-}$ mice infused with Ang II for 3 days. (D) Quantification of the number of bone marrow neutrophils from WT and $\mathrm{Lnk}^{-1-}$ mice at baseline and after 3 days of Ang II infusion that migrated to the bottom chambers of a transwell assay $(n=4-10)$. (E) Representative gelatin zymogram of MMP-9 activity in the supernatant of cultured neutrophils isolated from the bone marrow of WT and $L n k^{-1-}$ mice and activated ex vivo. Quantification is expressed as fold increase relative to WT $(n=4-5)$. Data are expressed as box-and-whisker plots (B and E) or mean \pm SEM (D). ${ }^{*} P<0.05,{ }^{* *} P<0.01,{ }^{* * *} P$ $<0.0001$ by 2-way ANOVA (B and $\mathbf{D}$ ) or 1-sided Student's $t$ test (E).

\section{Discussion}

$\mathrm{AD}$ is a devastating and often fatal condition for which optimal medical therapies are lacking, and surgical interventions pose a high risk. Elucidating early mechanisms of this disease is thus critical to develop effective preventative and therapeutic strategies. In this study, we demonstrate what we believe is a novel role for LNK in the pathology of AD in both experimental animals and humans. Importantly, we show that loss of LNK leads to early structural and inflammatory changes in the aortic wall, prior to the development of dissection, including reduced energy storage potential, elastin fragmentation, collagen degradation, increased neutrophil accumulation, and enhanced MMP-9 activity.

Besides inherited connective tissue diseases and familial aortic syndromes, hypertension and atherosclerosis are major risk factors for AD. Interestingly, the gene SH2B3 that encodes LNK has been shown to be associated with hypertension and coronary artery disease in multiple GWAS (4). Moreover, we and 
A $\operatorname{Lnk}^{-1-}$

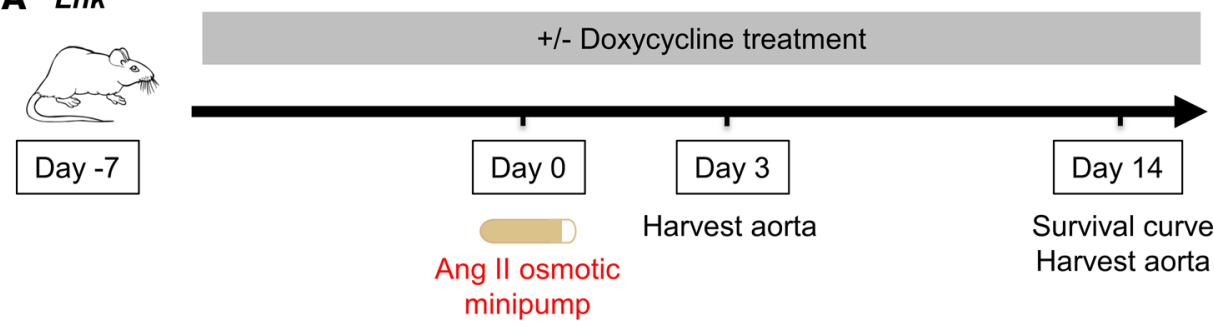

B

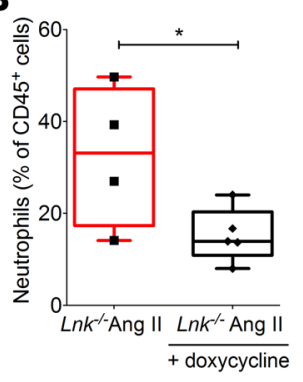

D

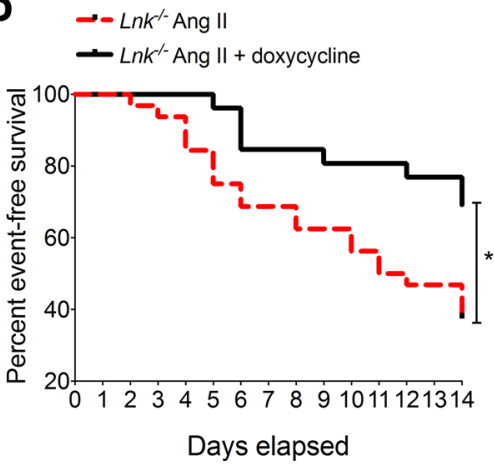

C

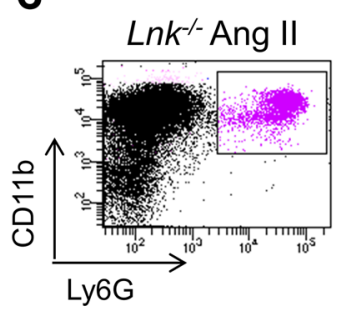

Lnk $k^{-1-}$ Ang II + doxycycline

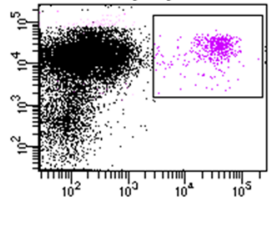

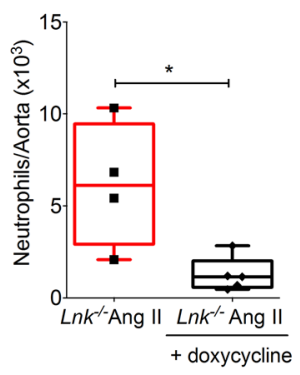

$\mathbf{E}$
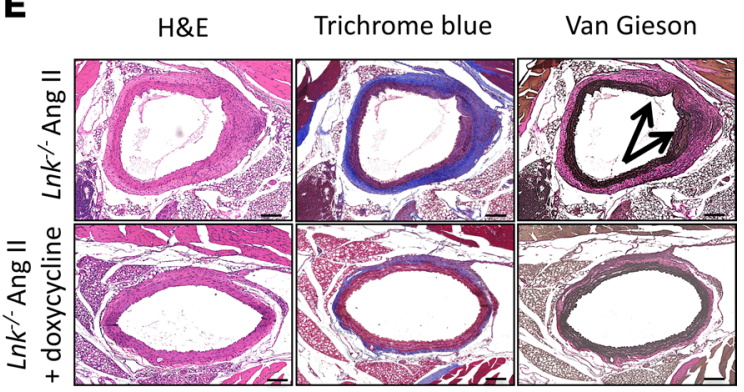

Figure 6. Doxycycline prevents the early recruitment of neutrophils into the aorta and markedly reduces the development of AD and rupture in $\mathbf{L n k}^{-/-}$mice. (A) Experimental design for $\mathrm{Lnk}^{-/-}$mice treated or not with doxycycline and infused with Ang II. (B) Flow cytometric quantification of the percentage of circulating neutrophils (CD45 ${ }^{+}$CD11 $\mathrm{b}^{+}{ }^{2} \mathrm{LCC}^{+}$ cells), and (C) representative flow cytometry dot plots for aortic neutrophils with quantification shown on the right at day 3 of Ang II infusion ( $n=4-5)$. (D) Kaplan-Meier survival curves during 14 days of Ang II infusion $(n=26-32)$. Results for the $\mathrm{Lnk}^{-1-}$ Ang II group is the sum of all experiments (initially presented in Figure 1 ) and shown again here for comparison. (E) Representative transverse sections of abdominal aorta stained with H\&E, Masson's trichrome blue, and Elastica van Gieson after 14 days of Ang II infusion. Arrows depict site of elastin degradation. Data are expressed as box-and-whisker plots. ${ }^{*} P<0.05$ by 2 -sided Student's $t$ test (B and $\mathbf{C}$ ) or by Mantel-Cox test (D).

others demonstrated that loss of LNK promotes hypertension and atherosclerosis in experimental animals $(5,12)$. Thus, it is interesting to speculate that genetic variation in $S H 2 B 3$ may provide a common link between hypertension, atherosclerosis, and $\mathrm{AD}$. We used PrediXcan to estimate the component of $S H 2 B 3$ gene expression determined by an individual's genetic profile and then correlated imputed gene expression with ICD9 codes containing dissection of aorta and/or dissecting aneurysm. In a cohort of more than 23,000 individuals of European descent, we found that increased expression of $S H 2 B 3$ was associated with a significant reduction in AD-related phenotypes (Figure 7).

Inflammation is increasingly recognized as playing a critical role in the development of $\mathrm{AD}$. For example, plasma levels of inflammatory markers such as $\mathrm{C}$-reactive protein, brain natriuretic peptide, and D-dimer are increased in patients with $\mathrm{AD}$ and correlate with a poor prognosis (3). In addition, imaging with ${ }^{18} \mathrm{~F}$-fluoro-deoxy-glucose (FDG) PET/CT, which detects vascular inflammation, showed that a greater uptake of ${ }^{18} \mathrm{~F}$-FDG was associated with increased risk of progression of the disease (13). Furthermore, increased neutrophils, macrophages, and MMP-9 have been detected in aortic tissue or blood of patients with AD (14-17). MMP-9, which degrades elastin and collagens, can be produced by neutrophils, macrophages, or vascular 


\section{PrediXcan}

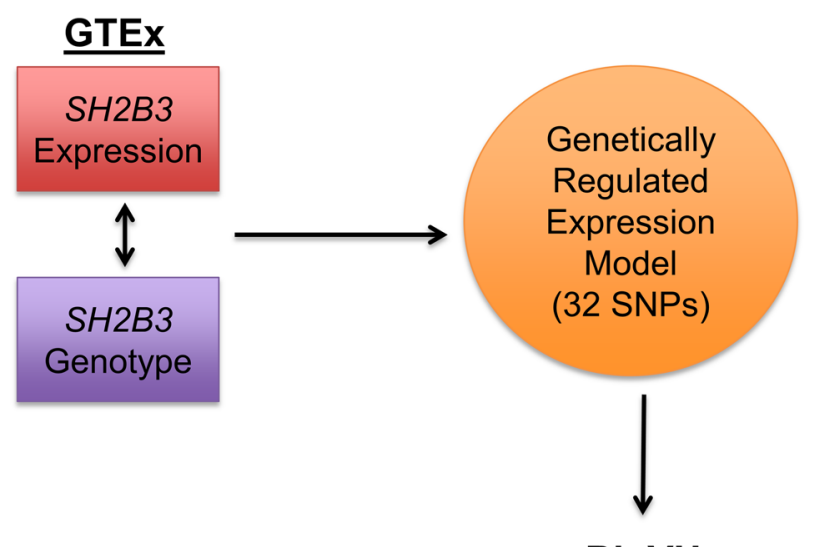

\section{BioVU}
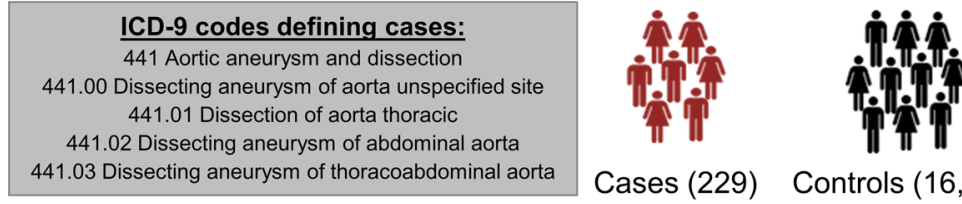

Controls $(16,965)$

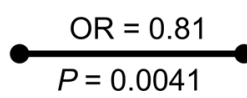

Figure 7. PrediXcan reveals association between increased SH2B3 expression and reduced risk of AD phenotypes in humans. Genotype-Tissue Expression (GTEx) was used to identify a model of 32 SNPs that predicted increased SH2B3 expression. This model was applied to Vanderbilt's BioVU cohort with the AD phenotype cases defined as containing at least one of the ICD9 codes listed on the left. The genetic model predicting increased SH2B3 expression was associated with decreased risk of the AD phenotype with the odds ratio (OR) and $P$ value shown.

smooth muscle cells $(16,18)$. Our studies using adoptive transfer of WT or LNK-deficient leukocytes into Rag $1^{-/-}$mice demonstrate that loss of LNK in immune cells plays a key role in development of AD (Figure 4) and that $L n k^{-/}$neutrophils secrete more MMP-9 when activated ex vivo and exhibit increased migratory capacity in the setting of Ang II infusion (Figure 5, D and E). RNA sequencing and pathway analysis further supports alterations in immune-related pathways in $L n k^{-/}$aortas following Ang II infusion (Supplemental Figure 4). Nevertheless, we cannot rule out a concomitant role of vascular LNK in this disease. To attempt to address the role of vascular LNK, we performed bone marrow transplantation studies in which WT or $L n k^{-/}$hematopoietic cells were transplanted into irradiated WT or $L n k^{-/}$mice. Unfortunately, all groups, including $L n k^{-/}$hematopoietic cells into $L n k^{-/}$mice, resulted in protection from $\mathrm{AD}$ (data not shown), suggesting that the irradiation itself altered the aortic matrix and conferred protection from $\mathrm{AD}$. Future studies utilizing cell-specific deletion of LNK will be helpful to determine the precise cell types in which LNK signaling contributes to $\mathrm{AD}$ and rupture.

LNK inhibits growth factor and cytokine receptor-mediated signaling in hematopoietic cells and endothelial cells through multiple mechanisms. LNK can directly interact with JAK2 to negatively regulate PI3K/Akt or ERK1/2 MAPK signaling pathways (4). Recently, it has been shown that PI3K is involved in MMP-9 activation and reactive oxygen species production in neutrophils (19). Thus, one mechanism by which loss of LNK may promote structural degradation is through increased PI3K activity and enhanced MMP-9 production by neutrophils.

Most of the current information about immunological changes in human $\mathrm{AD}$ is based on analyses of blood and aortic tissue after the dissection has occurred. Thus, an experimental model such as the Lnk mouse provides an opportunity to investigate causal changes that precede dissection. Other models of primarily thoracic $\mathrm{AD}$ involve genetic or pharmacological inhibition of matrix/structural proteins or the transforming growth factor $\beta$ pathway (20). In one such model, Ang II is infused following chronic administration 
of $\beta$-aminopropionitrile monofumarate (BAPN), a lysyl oxidase inhibitor that disrupts collagen and elastin crosslinking. A limitation of this model is that BAPN artificially disrupts aortic structural integrity. Thus, while this model may be useful to investigate mechanisms of $\mathrm{AD}$ propagation and rupture, it is insufficient to elucidate mechanisms that might initiate the matrix degradation. The most common animal model of abdominal $\mathrm{AD}$ involves Ang II infusion into hyperlipidemic $\mathrm{ApoE}^{-/-}$mice, in which dissection precedes aneurysm formation and atherosclerosis (21). Ang II infusion into $L n k^{-1-}$ mice provides a novel, simple, and rapid model of $\mathrm{AD}$ and rupture, without the confounding effects of hyperlipidemia or artificial disruption of aortic structural integrity, that recapitulates many features of the human disease including male predominance. Thus, this model provides a valuable tool to investigate mechanisms of disease, including sex differences, identify potential biomarkers of disease activity, and test new therapeutic strategies.

We found that inhibition of neutrophil migration and MMP activity with doxycycline dramatically reduced the incidence and progression of disease (Figure 6). Of note, the incidence of AD did not return to WT levels, suggesting that either we did not achieve complete inhibition of neutrophil migration and MMP activity or that neutrophil- and MMP-independent mechanisms might also be involved. To our knowledge, doxycycline has not been studied in the context of $\mathrm{AD}$, but rather in humans and animal models of aortic aneurysm with mixed results (22-25). Since circulating neutrophil-to-lymphocyte ratio and MMP-9 are elevated in patients with acute $\mathrm{AD}$ compared with patients with chronic aneurysm and healthy controls $(16,26)$, it is possible that doxycycline, or related drugs, may be more effective in the management of $\mathrm{AD}$ compared with aortic aneurysms. Future clinical trials in humans are needed to determine if doxycycline could represent a new therapy to prevent the development of $\mathrm{AD}$ in high-risk individuals or slow progression of disease.

In conclusion, our study highlights a key role for LNK in the pathology of AD in animals and humans. Furthermore, we describe an experimental model that can be used to investigate mechanisms of AD and test new therapeutic strategies, with the potential to inform both inherited and acquired forms of this disease.

\section{Methods}

Animals and BP measurement. C57BL/6J WT mice and Rag1-/- mice were purchased from Jackson Laboratories. $L n k^{-1-}$ on a $\mathrm{C} 57 \mathrm{BL} / 6 \mathrm{~J}$ background were generated as previously described $(5,27)$. Unless otherwise indicated, male mice were used for these studies. At approximately 8 to 12 weeks of age, osmotic minipumps (Alzet, DURECT Corporation, model 2002) were implanted subcutaneously for infusion of Ang II (1,200 $\mathrm{ng} / \mathrm{kg} / \mathrm{min}$ ) or vehicle (sodium chloride/acetic acid solution) for 3 days or 14 days depending on the experiment. BP was measured noninvasively using tail cuff or invasively using carotid radiotelemetry as previously described (28). After telemetry placement, mice were allowed to recover for 10 days prior to osmotic mini-pump implantation. Mice were euthanized using $\mathrm{CO}_{2}$ inhalation at the end of the planned experiment or if hindlimb paralysis (sign of vascular insufficiency due to AD) was observed.

Mouse serum collection and cholesterol measurements. Mice were fasted overnight and euthanized before blood collection. Blood was collected from the left and right ventricles and centrifuged at 3,000 $g$ for 15 minutes. Serum was collected from the centrifuged samples and total cholesterol was measured by the Translational Pathology Shared Resource core at Vanderbilt University Medical Center.

PrediXcan analysis. BioVU is a large biobank of Vanderbilt University Medical Center (VUMC) that houses deidentified DNA samples linked to phenotypic data derived from the electronic medical record (EMR) system (29). DNA samples were genotyped on the Multi-Ethnic Global Array, and the genotypes were imputed into the Haplotype Reference Consortium reference panel using the Michigan imputation server. Imputed data and the 1000 Genome Project data of CEU, YRI, and CHB+JPT descent were combined to carry out principal component analysis (PCA) and only samples of European descent were extracted for analysis based on the PCA plot. Expression of $\mathrm{SH} 2 \mathrm{~B} 3$ across multiple tissues was imputed for each person based on their genotypes using the expression predictors previously trained on the GTEx release of RNA sequencing data (10). Logistic regression was performed for a binary AD phenotype as the outcome and tissue-specific predicted gene expression as the risk factor while adjusting for covariates of age, gender, genotyping array type/batch, and 3 principle components of ancestry. The AD phenotype was defined as containing at least one of the following ICD9 codes: 441, 441.00, 441.01, 441.02, 441.03; and the controls were defined as not having any diseases of the arteries, arterioles, and capillaries (i.e., excluding phecodes 440-449.99; https://phewascatalog.org/phecodes). The combination of 32 SNPs that predict SH2B3 expression and associated with the AD phenotype are shown in Supplemental Table 1. This expression predictor explains $8 \%$ of the measured expression in the brain nucleus accumbens basal ganglia of GTEx. 
Contrast-enhanced in vivo microCT imaging. Mice were injected intravenously every 2 days with $100 \mu 1$ of 15-nm AuroVist gold nanoparticles (Nanoprobes). Under isoflurane anesthesia, microCT images of the abdominal cavity of WT and $L n k^{-/}$mice were obtained prior to Ang II treatment and during Ang II infusion, as indicated in the figures using a vivaCT80 (Scanco Medical AG). Axial images were acquired with 62.4- $\mu \mathrm{m}^{3}$ isotropic voxels, in a 31.9-mm field of view, with acquisition settings of 2,000 projections/rotation, $200 \mathrm{msec}$ integration, $45 \mathrm{kVp}$, and $177 \mu \mathrm{A}$. Total radiation exposure time was 6 minutes 40 seconds. The volume of interest was defined by loosely outlining the perimeter of the abdominal aorta from the superior to the inferior mesenteric arterial branches, then applying a 3D Gaussian noise filter and visually optimized attenuation threshold to create a $3 \mathrm{D}$ reconstruction of the contrast-enhanced blood pool. The mean thickness of the abdominal aorta reconstruction was determined using the $3 \mathrm{D}$ distance transformation method.

Immunohistochemistry. After euthanasia, mice were perfusion-fixed at physiological pressure with $10 \%$ neutral buffered formalin. Aortas were incubated 24 hours in Decalcifying Solution-Lite (Sigma-Aldrich), embedded in paraffin, and cut in cross sections of $5 \mu \mathrm{m}$. Slides were deparaffinized and heat- or enzyme-induced antigen retrieval was performed. Slides were incubated with anti-CD3 (ab16669, Abcam) for T cells, anti-neutrophil marker (ab2557, Abcam) for neutrophils, anti-CD45R (550286, BD Pharmingen) for B cells, and anti-F4/80 (NB600-404, Novus Biologicals) for macrophages. The Bond Polymer Refine detection system (DS9800, Leica) was used for visualization. Slides were then dehydrated, cleared, and coverslipped.

Histological staining. Sections of $10 \mu \mathrm{m}$ were stained with hematoxylin and eosin, Masson's trichrome, picrosirius red, or Elastica van Gieson to assess extracellular matrix remodeling. Sections were digitized with Leica SCN400 slide scanner. Stained areas were determined using color-based thresholding and quantified by Digital Image Hub software (4.0.6). To assess collagen fibers, picrosirius red-stained sections were studied under polarized light and collagen fibers were quantified using custom feature-based panoramic image stitching and color segmentation algorithms in MATLAB (2016a, MathWorks) as previously described (30).

Mechanical testing. Biaxial mechanical tests were performed on excised segments of the aorta using methods described previously (31). Four segments of the aorta — ascending, proximal descending thoracic, suprarenal abdominal, and infrarenal abdominal - from WT and $L n k^{-1-}$ mice infused for 3 days with vehicle or Ang II were gently cleaned of excess perivascular tissue and all branches were ligated. Vessels were then cannulated with custom glass pipettes and mounted within a custom computer-controlled testing device (32). After an equilibration period of 15 minutes in heated Hanks balanced salt solution $\left(37^{\circ} \mathrm{C}\right.$, GIBCO 14025134$)$, the vessels were preconditioned via 4 cycles of pressurization (from 10 to $140 \mathrm{mmHg}$ ) at the in vivo axial length. The unloaded geometry (outer diameter, wall thickness, axial length) was recorded and the different segments were exposed to 3 cyclic pressure-diameter $(P-d)$ protocols at 3 different fixed axial lengths and 4 cyclic axial force-length $(f-l)$ tests at 4 different fixed intraluminal pressures. The biaxial data (outer diameter, luminal pressure, axial length, axial force) were collected online and used in a nonlinear regression analysis to identify best-fit values of the material parameters in a validated nonlinear constitutive relation using a previously described algorithm (MATLAB) (31). Biaxial wall stress, intrinsic material stiffness, and stored strain energy were calculated for in vivo loading conditions (e.g., group-specific in vivo axial stretch and systolic pressure). Note that the experimentally determined systolic pressures were $124 \mathrm{mmHg}$ for sham and $151 \mathrm{mmHg}$ for Ang II-infused mice.

$q R T-P C R$ analysis. Aortas were homogenized in TRIzol using Lysing Matrix D tubes (MP Biomedicals) and a MiniBeadBeater homogenizer (BioSpec). Lysates were then subjected to phenol-chloroform extraction. RNA was subsequently isolated from the aqueous phase using an RNeasy Mini Kit (Qiagen) according to the manufacturer's instructions. cDNA was made using a High-Capacity cDNA Reverse Transcription kit (Applied Biosystems) according to the manufacturer's instructions. A Taqman assay system and probes (Thermo Fisher Scientific) were used to detect Coll1a1, Coll3a1, MMP-2, MMP-9, TIMP-1, and TIMP-2. A Taqman assay system and probe for GAPDH (Thermo Fisher Scientific) was used as an endogenous control. Relative quantification was determined using the comparative CT method with data normalized to GAPDH and calibrated to the average of the control group.

RNA sequencing. Total RNA was extracted with the TRIzol reagent. RNA quality was assessed using the 2100 Bioanalyzer (Agilent). At least $200 \mathrm{ng}$ of DNase-treated total RNA with a RNA integrity number greater than 6 was used to generate ribo-depleted libraries using TruSeq Stranded RNA sample kits with indexed adaptors (Illumina). Library quality was assessed using the 2100 Bioanalyzer and libraries were quantified using KAPA Library Quantification Kits (KAPA Biosystems). Pooled libraries were subjected to 75-bp paired-end sequencing according to the manufacturer's protocol (Illumina HiSeq 3000). Bcl2fastq2 Conversion Software (Illumina) was used to generate demultiplexed Fastq files. 
For the analysis, after adapter trimming by cutadapt (33), RNA sequencing reads were aligned to the mouse reference genome mm10 using STAR (34) and quantified by featureCounts (35). Differential analyses were performed by DESeq2 (36). The significantly changed genes were assessed with adjusted $P \leq 0.05$ and absolute fold change $\geq 1.5$. To visualize the expression pattern of differential genes, cluster analysis and PCA were performed using R software (www.r-project.org/). To analyze function changes between 2 groups of mice, pathway analysis was performed with WebGestalt software (37) for genes with $P$ value $\leq 0.05$ and absolute fold change $\geq 1.5$.

Zymography. The zymography protocol was performed as described previously (38). Aortic proteins were extracted in a cold lysis buffer $(0.025 \mathrm{M}$ Tris- $\mathrm{HCl}, 0.1 \mathrm{M} \mathrm{NaCl}, 1 \% \mathrm{v} / \mathrm{v}$ Nonidet P-40, protease inhibitor cocktail, $\mathrm{pH}$ 7.5). Samples were standardized for protein concentration and run in a $10 \%$ polyacrylamide gel containing $0.1 \% \mathrm{w} / \mathrm{v}$ gelatin. After electrophoresis, gels were renatured $(2.5 \%$ Triton X-100) for 30 minutes at room temperature and incubated at $37^{\circ} \mathrm{C}$ overnight in developing buffer $(0.05 \mathrm{M}$ Tris- $\mathrm{HCl}$, $0.2 \mathrm{M} \mathrm{NaCl}, 0.005 \mathrm{M} \mathrm{CaCl}_{2}, 0.25 \%$ Triton X-100, $\mathrm{pH}$ 7.8). After staining the gels with $0.5 \%$ Coomassie brilliant blue R-250 for 1 hour, the gels were destained until areas of gelatinolytic activity appeared as clear sharp bands. The molecular sizes of gelatinolytic activities were determined with the use of protein standards (Precision Plus Protein, Bio-Rad Laboratories). Band intensity was quantified using Image Lab3 software. MMP activity was displayed as fold change compared with the WT sham group. For each experiment the average of the WT sham group was set at 1 .

Flow cytometry. Single-cell suspensions of aorta were prepared as previously described (39). Briefly, aortas with surrounding perivascular fat were minced and digested with $1 \mathrm{mg} / \mathrm{ml}$ collagenase $\mathrm{A}, 1 \mathrm{mg} / \mathrm{ml}$ collagenase B, and $100 \mu \mathrm{g} / \mathrm{ml}$ DNAse I in RPMI 1640 medium supplemented with $5 \%$ FBS for 30 minutes at $37^{\circ} \mathrm{C}$. Tissue homogenates were filtered through a $40-\mu \mathrm{m}$ cell strainer. Antibodies used for flow cytometry were as follows: CD45-APC (clone 30-F11, BioLegend), CD11b-APCCy7 (clone M1/70, BioLegend), Ly6G-PE (clone 1A8, BioLegend), F4/80-FITC (clone BM8, BioLegend), CD3-PerCPCy5.5 (clone 17A2, BioLegend), CD19-FITC (clone 6D5, BioLegend), CD206-PE (clone C068C2, BioLegend), and CD80-PECy7 (clone 16-10A1, BioLegend). For live/dead staining, LIVE/DEAD Fixable Violet Dead Cell Stain (Life Technologies) was used. Aortic cell numbers were quantified using 123count eBeads (eBioscience). Flow cytometric multiparameter acquisition was performed on a BD FACSCanto II system and data were analyzed with BD FACSDiva software (BD Biosciences). Gates were set using fluorescence-minus-one controls.

Adoptive transfer. Spleens of WT or $L n k^{-1-}$ mice were flushed with cold PBS and erythrocytes were eliminated using RBC lysis buffer. To fully reconstitute immune niches including peritoneal B1 cells, $2 \times$ $10^{7}$ splenic $\mathrm{CD} 45^{+}$leukocytes were mixed with $5 \times 10^{5}$ peritoneal B cells isolated using B cell isolation kit (Miltenyi Biotec) from either WT or $L n k^{-/}$mice and injected intraperitoneally into recipient $R a g 1^{-/-}$mice. Two weeks after injection, recipient $\mathrm{Rag}^{1^{-/-}}$mice were subjected to Ang II infusion for 2 weeks.

Doxycycline treatment. Lnk $^{-/-}$mice were treated with doxycycline $(0.44 \mathrm{mg} / \mathrm{ml}$, Sigma-Aldrich $)$ starting 1 week prior to Ang II infusion and continuing during the 2 weeks of Ang II infusion. Mice received a calculated dose of $100 \mathrm{mg} / \mathrm{kg} /$ day in the drinking water as previously described (40). Each bottle was protected from the light and fresh doxycycline solution was prepared every 2 days.

Neutrophil isolation from the bone marrow. Mouse bone marrow cells were harvested by flushing the marrow of the femurs with RPMI supplemented with 10\% FBS. Neutrophils were isolated using Histopaque-based density gradient centrifugation as previously described (41).

Chemotaxis assay. This assay was performed using the HTS Transwell 24-well plates/3- $\mu$ m pore (Corning). Neutrophils isolated from the bone marrow were resuspended with RPMI/1\% FBS. Cells $\left(1 \times 10^{5}\right)$ were resuspended in $100 \mu \mathrm{l}$ of RPMI/1\% FBS and added into the upper wells. CXCL2 (100 ng/ml) was added into the lower wells. The plates were incubated at $37^{\circ} \mathrm{C}$ with $5 \% \mathrm{CO}_{2}$ for 1 hour. The lower cell suspensions were analyzed by flow cytometry to quantify the number of migrated neutrophils.

Ex vivo neutrophil activation. Neutrophils isolated from the bone marrow of WT and Lnk ${ }^{-1}$ mice were resuspended in RPMI/0\% FBS and placed in 96-well plates (50,000 neutrophils/100 $\mu$ l) with PMA and ionomycin (cell activation cocktail, BioLegend). Cells were incubated at $37^{\circ} \mathrm{C}$ with $5 \% \mathrm{CO}_{2}$ for 3 hours. The media were collected for gelatin zymography analysis.

Mouse chemokine array. The mouse chemokine array kit (Proteome Profiler Array, catalog ARY020, R\&D Systems) was used for the parallel determination of relative levels of selected mouse chemokines in the aortas of WT and $L n k^{-1}$ mice infused with Ang II for 3 days. The array was performed according to the manufacturer's instructions. 
Statistics. Data were analyzed using Student's $t$ tests (for 2-group comparisons), 2-way ANOVA (for multiple group comparisons), or Mantel-Cox test (for survival analysis) and are presented as box-and-whisker plots or mean \pm SEM as indicated. $P<0.05$ was considered significant.

Study approval. All animal procedures were approved by the Vanderbilt IACUC.

\section{Author contributions}

FL, AK, MRB, DSP, JAC, JDH, and MSM designed the experiments. FL, AK, MRB, MRA, XZ, JPVB, YC, LX, MAS, WGM, KAG, BLD, SZ, YG, and DSP generated and analyzed data. YS, DSP, NJC, JAC, $\mathrm{JDH}$, and MSM supervised the study and analyzed data. FL, AK, MRB, MRA, XZ, SZ, JAC, JDH, and MSM wrote the manuscript and revised it for intellectual content.

\section{Acknowledgments}

We acknowledge the Vanderbilt Translational Pathology Shared Resource (NCI/NIH Cancer Center 5P30 CA68485-19), Mouse Metabolic Phenotyping Center (DK59637), Technologies for Advanced Genomics Core (P30 CA68485), and the Institute of Imaging Science (NIH grant S10OD016204 to DSP). This work was supported by an American Heart Association postdoctoral fellowship award (16POST29950007) to FL, an NIH U01 award (HL116323) to JDH, and an NIH NHLBI K08 award (HL121671), a Gilead Cardiovascular Scholars Grant, and an NIH DP2 award (HL137166) to MSM.

Address correspondence to: Meena S. Madhur, Department of Medicine, Division of Clinical Pharmacology, 2215 Garland Avenue, P415D MRB IV, Vanderbilt University, Nashville, Tennessee 37232, USA. Phone: 615.875.3273; Email: meena.madhur@vanderbilt.edu.

MAS's present address is: Department of Pharmacology and Toxicology, Mansoura University, Mansoura, Egypt.

YG's present address is: Bioinformatics Shared Resource, University of New Mexico Comprehensive Cancer Center, Albuquerque, New Mexico, USA.

1. Goldfinger JZ, Halperin JL, Marin ML, Stewart AS, Eagle KA, Fuster V. Thoracic aortic aneurysm and dissection. $J$ Am Coll Cardiol. 2014;64(16):1725-1739.

2. Nienaber CA, et al. Gender-related differences in acute aortic dissection. Circulation. 2004;109(24):3014-3021.

3. Luo F, Zhou XL, Li JJ, Hui RT. Inflammatory response is associated with aortic dissection. Ageing Res Rev. 2009;8(1):31-35.

4. Devallière J, Charreau B. The adaptor Lnk (SH2B3): an emerging regulator in vascular cells and a link between immune and inflammatory signaling. Biochem Pharmacol. 2011;82(10):1391-1402.

5. Saleh MA, et al. Lymphocyte adaptor protein LNK deficiency exacerbates hypertension and end-organ inflammation. J Clin Invest. 2015;125(3):1189-1202.

6. Zhang X, Shen YH, LeMaire SA. Thoracic aortic dissection: are matrix metalloproteinases involved? Vascular. 2009;17(3):147-157.

7. Takeshita S, et al. Modulation of oxidative burst of neutrophils by doxycycline in patients with acute myocardial infarction. J Antimicrob Chemother. 2002;49(2):411-413.

8. Moon A, Gil S, Gill SE, Chen P, Matute-Bello G. Doxycycline impairs neutrophil migration to the airspaces of the lung in mice exposed to intratracheal lipopolysaccharide. J Inflamm (Lond). 2012;9(1):31.

9. Prall AK, et al. Doxycycline in patients with abdominal aortic aneurysms and in mice: comparison of serum levels and effect on aneurysm growth in mice. J Vasc Surg. 2002;35(5):923-929.

10. Gamazon ER, et al. A gene-based association method for mapping traits using reference transcriptome data. Nat Genet. 2015;47(9):1091-1098.

11. GTEx Consortium. Human genomics. The Genotype-Tissue Expression (GTEx) pilot analysis: multitissue gene regulation in humans. Science. 2015;348(6235):648-660.

12. Wang W, et al. LNK/SH2B3 loss of function promotes atherosclerosis and thrombosis. Circ Res. 2016;119(6):e91-e103.

13. Kato K, Nishio A, Kato N, Usami H, Fujimaki T, Murohara T. Uptake of 18F-FDG in acute aortic dissection: a determinant of unfavorable outcome. J Nucl Med. 2010;51(5):674-681.

14. Wu D, et al. Inflammatory cell infiltrates in acute and chronic thoracic aortic dissection. Aorta (Stamford). 2013;1(6):259-267.

15. del Porto F, et al. Inflammation and immune response in acute aortic dissection. Ann Med. 2010;42(8):622-629.

16. Kurihara T, et al. Neutrophil-derived matrix metalloproteinase 9 triggers acute aortic dissection. Circulation. 2012;126(25):3070-3080

17. Del Porto F, et al. The multitasking role of macrophages in Stanford type A acute aortic dissection. Cardiology. 2014;127(2):123-129.

18. Cifani N, et al. Stanford-A acute aortic dissection, inflammation, and metalloproteinases: a review. Ann Med. 2015;47(6):441-446.

19. Gupta V, et al. The effect of phosphatidylinositol-3 kinase inhibition on matrix metalloproteinase-9 and reactive oxygen species release from chronic obstructive pulmonary disease neutrophils. Int Immunopharmacol. 2016;35:155-162.

20. Michel JB, Jondeau G, Milewicz DM. From genetics to response to injury: vascular smooth muscle cells in aneurysms and 
dissections of the ascending aorta. Cardiovasc Res. 2018;114(4):578-589.

21. Saraff K, Babamusta F, Cassis LA, Daugherty A. Aortic dissection precedes formation of aneurysms and atherosclerosis in angiotensin II-infused, apolipoprotein E-deficient mice. Arterioscler Thromb Vasc Biol. 2003;23(9):1621-1626.

22. Abdul-Hussien H, Hanemaaijer R, Verheijen JH, van Bockel JH, Geelkerken RH, Lindeman JH. Doxycycline therapy for abdominal aneurysm: Improved proteolytic balance through reduced neutrophil content. J Vasc Surg. 2009;49(3):741-749.

23. Curci JA, et al. Preoperative treatment with doxycycline reduces aortic wall expression and activation of matrix metalloproteinases in patients with abdominal aortic aneurysms. J Vasc Surg. 2000;31(2):325-342.

24. Meijer CA, et al. Doxycycline for stabilization of abdominal aortic aneurysms: a randomized trial. Ann Intern Med. 2013;159(12):815-823.

25. Iida Y, et al. Efficacy and mechanism of angiotensin II receptor blocker treatment in experimental abdominal aortic aneurysms. PLOS ONE. 2012;7(12):e49642.

26. Sbarouni E, Georgiadou P, Analitis A, Voudris V. High neutrophil to lymphocyte ratio in type A acute aortic dissection facilitates diagnosis and predicts worse outcome. Expert Rev Mol Diagn. 2015;15(7):965-970.

27. Takaki S, et al. Control of B cell production by the adaptor protein lnk. Definition Of a conserved family of signal-modulating proteins. Immunity. 2000;13(5):599-609.

28. Madhur MS, et al. Interleukin 17 promotes angiotensin II-induced hypertension and vascular dysfunction. Hypertension. 2010;55(2):500-507

29. Roden DM, et al. Development of a large-scale de-identified DNA biobank to enable personalized medicine. Clin Pharmacol Ther. 2008;84(3):362-369.

30. Bersi MR, Khosravi R, Wujciak AJ, Harrison DG, Humphrey JD. Differential cell-matrix mechanoadaptations and inflammation drive regional propensities to aortic fibrosis, aneurysm or dissection in hypertension. JR Soc Interface. 2017;14(136):20170327.

31. Ferruzzi J, Bersi MR, Humphrey JD. Biomechanical phenotyping of central arteries in health and disease: advantages of and methods for murine models. Ann Biomed Eng. 2013;41(7):1311-1330.

32. Gleason RL, Gray SP, Wilson E, Humphrey JD. A multiaxial computer-controlled organ culture and biomechanical device for mouse carotid arteries. J Biomech Eng. 2004;126(6):787-795

33. Martin M. Cutadept removes adapter sequences from high-throughput sequencing reads. EMBnetjournal. 2011;17(1):10-12.

34. Dobin A, et al. STAR: ultrafast universal RNA-seq aligner. Bioinformatics. 2013;29(1):15-21.

35. Liao Y, Smyth GK, Shi W. featureCounts: an efficient general purpose program for assigning sequence reads to genomic features. Bioinformatics. 2014;30(7):923-930.

36. Love MI, Huber W, Anders S. Moderated estimation of fold change and dispersion for RNA-seq data with DESeq2. Genome Biol. 2014;15(12):550.

37. Wang J, Vasaikar S, Shi Z, Greer M, Zhang B. WebGestalt 2017: a more comprehensive, powerful, flexible and interactive gene set enrichment analysis toolkit. Nucleic Acids Res. 2017;45(W1):W130-W137.

38. Toth M, Sohail A, Fridman R. Assessment of gelatinases (MMP-2 and MMP-9) by gelatin zymography. Methods Mol Biol. 2012;878:121-135.

39. Laroumanie F, Dale BL, Saleh MA, Madhur MS. Intracellular staining and flow cytometry to identify lymphocyte subsets within murine aorta, kidney and lymph nodes in a model of hypertension. J Vis Exp. 2017;(119):e55266.

40. Pyo R, et al. Targeted gene disruption of matrix metalloproteinase-9 (gelatinase B) suppresses development of experimental abdominal aortic aneurysms. J Clin Invest. 2000;105(11):1641-1649.

41. Swamydas M, Luo Y, Dorf ME, Lionakis MS. Isolation of mouse neutrophils. Curr Protoc Immunol. 2015;110:3.20.1-3.20.15. 